about their child's care in order to improve patient outcomes.

Aim We aim to improve the satisfaction with information provision regarding CNLD and Home Oxygen requirements, to a self-evaluated level of $4-5 / 5$, for at least $80 \%$ of parents with affected children at the William Harvey Hospital (WHH) by March 2019.

Methods Continuous questionnaires were answered by parents of CNLD patients at WHH to quantify the effect of our PSDA cycles. A total of four cycles were conducted which involved distributing questionnaires to healthcare professionals, composing and delivering a parent reviewed information leaflet and creating a parent information video and finally presenting our evidence and parent information resources as an intervention to neonatal respiratory team at WHH.

Results When measuring parental understanding of CNLD, self-reported rates of 4 or $5 / 5$ increased from $64 \%$ to $100 \%$ by the completion of our project. Similarly, self-reported rates of 4 or $5 / 5$ for parent satisfaction with the provision of information regarding CNLD and Home Oxygen rose from $64 \%$ and $82 \%$ to $100 \%$ respectively. Combined effect of written (leaflet) and audio-visual (video) information were thought to contribute to the high level of satisfaction in parents and carers.

Conclusion Parental understanding of CNLD is vital in order to provide the best care for affected infants. Care-pathways must ensure high standards are maintained and we demonstrate this is achievable with clear parent information resources and education of the relevant healthcare team.

\section{G282(P) GASTROSTOMY PREVALENCE IN THE PAEDIATRIC POPULATION: A SYSTEMATIC REVIEW}

${ }^{1} \mathrm{R}$ Lau, ${ }^{1} \mathrm{~N}$ Kharma, ${ }^{2} \mathrm{C}$ Pardy, ${ }^{3} \mathrm{~V}$ Kelly, ${ }^{2}$ Y Yardley. ${ }^{1}$ Guy's, King's, and St. Thomas' School of Medical Education, King's College London, London, UK; ${ }^{2}$ Department of Paediatric Surgery, Evelina Children's Hospital, London, UK; ${ }^{3}$ Department of Paediatric Neurology, Evelina Children's Hospital, London, UK

\subsection{6/archdischild-2020-rcpch.245}

Aim It is known that the rate of gastrostomy insertion in the paediatric population is increasing. The prevalence of gastrostomies in the paediatric population has significant implications for health service provision, both for insertion and longterm management of the tubes. We sought to identify published literature describing the prevalence of gastrostomy tubes in the general paediatric population.

Methods A systematic review was performed using the keywords 'gastrostomy', 'paediatric', and 'prevalence' to search PubMed and Medline. The search was limited to 2008-2019. Duplicates and abstracts not containing the word 'gastrostomy' were excluded. The remaining abstracts were read. Full text versions of those relevant to the study were selected and the references scrutinised for any additional articles that were of interest to the study.

Results The initial search returned 3953 articles; 552 duplicates were excluded. The remaining 3401 articles were censored to those published in the last ten years (2008-2019), leaving 2060. 526 included the word 'gastrostomy', whose abstracts were read, leaving 15 papers focusing specifically on gastrostomy prevalence for which the full text was read. The references of these papers were scrutinised, yielding 1 additional publication, bringing our final count to 16 .
Of the 16 papers, only 8 were exclusively paediatric. Four of these were studies of population-wide period prevalence. Of these, three studied gastrostomy tube insertions (one solely PEG insertions), two report insertion rates across a population, but not the prevalence of indwelling gastrostomy tubes in that population. Only one study reported the prevalence of gastrostomy in a paediatric population. This found a rate of tube feeding at home of 83-92:100,000 children/year, of which $60 \%$ were gastrostomy tubes (50-55 gastrostomies/ 100,000 children/year).

Conclusion There is a significant volume of published literature describing the indications for, insertion techniques and complications of gastrostomy. However, literature relating to the prevalence of gastrostomy use in the paediatric population is scarce. Further studies are required reporting the prevalence of gastrostomies across a paediatric population, as this has significant resource implications and is of interest to policy makers, health care commissioners and providers.

\section{G283(P) APPRAISAL OF POST PERMANENCE CLINIC}

BJ Cooper, SM John-Legere. Evelina Community, Guys and St Thomas' NHS FT, London, UK

\subsection{6/archdischild-2020-rcpch.246}

Background The Adoption Support Fund (ASF) recognised additional needs of adopted children and those placed under a Special Guardianship Order (SGO) which has increased awareness and referral.

Aim To explore the feasibility and value of a specific clinic for children placed permanently with adopters or special guardians who have been referred to Community Paediatrics.

Method Clinic led by a clinician experienced in working with traumatised children. We looked at risks, presenting concerns (table 1), new diagnoses made, actions taken and parental satisfaction.

Results 29 children were referred from April 17- March 19: 12 on SGOs; 17 adopted. Referrals came from CAMHS, GPs, school and paediatricians. Average ages at permanent placement were the same for both groups - 3 years of age; average age at referral -6 years.

Increased ACEs including, child abuse and neglect, and antenatal risks, were very common.

$24 \%$ of children were born premature and $20 \%$ small for dates.

Pre-clinic diagnoses:

- 11 children known to CAMHS diagnosed with mixed emotional behavioural disorder and attachment issues

- 2 with language delay

Abstract 283(P) Table 1 Parental concerns

\begin{tabular}{lll}
\hline Concern & Primary Concern & Additional Concern \\
\hline Physical & 6 & 1 \\
Externalising Behaviours & 5 & 10 including 2 sexualised behaviour \\
Internalising Behaviours & 2 & 0 \\
Attention/?ADHD & 4 & 5 \\
Eating and Drinking & 3 & 3 \\
Elimination & 1 & 0 \\
Sleep & 2 & 0 \\
\hline
\end{tabular}

